\title{
EFFECT OF SEVERE DEFORMATION ON THE STRUCTURE, FRACTURE PATTERN AND MECHANICAL PROPERTIES OF REFRACTORY METALS
}

\author{
V. P. Pilyugin*, T. M. Gapontseva, T. P. Tolmachev, T. I. Chashchukhina, \\ L. M. Voronova, M. V. Degtyarev \\ M. N. Miheev Institute of Metal Physics, Ural Branch of the Russian Academy of Sciences, Ekaterin- \\ burg, 18 S. Kovalevskoy St., 620990, Russia \\ *Corresponding author. E-mail: pilyugin@imp.uran.ru \\ Address for correspondence: 18 S. Kovalevskoy St., Ekaterinburg, 620108, Russian Federation \\ Tel.: +7 (343) 3783805
}

High-pressure torsion is a method enabling one to obtain high strains without destroying the material being processed and to form the submicro- and nanocrystalline states in it. In this paper, this technique serves to process some brittle refractory metals. Particularly, the effect of pressure on the plasticity of brittle refractory metals strained by high pressure torsion using Bridgman anvils is studied. Namely, the critical pressure required for the realization of severe plastic deformation of refractory metals without destroying it by high pressure torsion is established. This value of the deformation pressure is estimated as 0.05 of the bulk modulus or 0.015 of the shear modulus of each metal. It has been established that the structural transformations of brittle metals caused by high pressure torsion forming the submicro- and nanocrystalline states have a significant effect on the mechanical properties of the metals as leading to significant work hardening. Moreover, with increasing strain (the number of anvil rotations), the mechanism of the fracture of the samples changes from cleavage to quasi-ductile one on the boundaries of the crystallites.

Keywords: structure, severe plastic deformation, high pressure, critical pressure, refractory metals, plasticity, nanostructure, brittle fracture, ductile fracture.

DOI: $10.17804 / 2410-9908.2017 .6 .112-119$

\section{References}

1. Drits M.E., ed. Element Properties: reference work. Moscow, Metallurgy Publ., 1985, 672 p. (In Russian).

2. $\quad$ Frost H.J., Ashby M.F. Deformation-mechanism maps. The plasticity and creeps of metals and ceramics. Cambridge University, UK, Pergamon press, 1982, $166 \mathrm{p}$.

3. Bridgman P.W. Effects of High Shearing Stress Combined with High Hydrostatic Pressure. Physical Review, 1935, vol. 48, pp. 825-847.

4. $\quad$ Pilyugin V.P., Voronova L.M., Degtyarev M.V., Chashchukhina T.I., Vykhodets V.B., Kurennykh T.E. Structure evolution of pure iron upon low-temperature deformation under high pressure. The Physics of Metals and Metallography, 2010, vol. 110, no. 6, pp. 564-573. DOI: $10.1134 / \mathrm{S} 0031918 X 10120070$

5. Pilyugin V.P., Gapontseva T.M., Chashchukhina T.I., Voronova L.M., Shchinova L.I., Degtyarev M.V. Evolution of the structure and hardness of nickel upon cold and low-temperature deformation under pressure. The Physics of Metals and Metallography, 2008, vol. 105, no. 4, pp. 409-419. DOI: 10.1134/S0031918X08040157

6. Degtyarev M.V., Chashchukhina T.I., Voronova L.M., Patselov A.M., Pilyugin V.P. Influence of the relaxation processes on the structure formation in pure metals and alloys under high-pressure torsion. Acta Materialia, 2007, vol. 55, no. 18, pp. 6039-6050. DOI: 10.1016/j.actamat.2007.04.017

7. Gapontseva T.M., Pilyugin V.P., Degtyarev M.V., Voronova L.M., Chashchukhina T.I., Patselov A.M. Structural Changes and Properties of Molybdenum upon Cold and Cryogenic 
Deformation under Pressure. Russian Metallurgy (Metally), 2014, no. 10, pp. 812-816. DOI: $10.1134 / \mathrm{S} 0036029514100024$

8. Gorbatov V.I., Polev V.F., Pilugin V.P., Korshunov I.G., Smirnov A.L., Talutz S.G., Brytkov D.A. Thermal diffusivity of submicro- and nanocrystalline niobium, titanium, and zirconium at high temperatures. High Temperature, 2013, vol. 51, no. 4, pp. 482-485. DOI: $10.1134 /$ S0018151X1304010X

9. Pilyugin V., Patselov A., Tolmachev T., Chernyshov E., Ancharov A., Brytkov D. The Influence of Nanocrystalline Structure of Iron on б-e Phase Transformations under Pressure. Material Science Forum, 2013, vol. 738-739, pp. 108-113. DOI: 10.4028/www.scientific.net/MSF.738-739.108

10. Koroleva L.F. Nanoparticulate zirconia-modified solid solutions of aluminum-iron oxides for polishing titanium metal. Diagnostics, Resource and Mechanics of materials and structures, 2015, iss. 1. Available at: http://dream-journal.org/DREAM_Issue_1_2015_Koroleva_L._F..pdf

11. Pilyugin V.P., Voronova L.M., Gapontseva T.M., Chashchukhina T.I., Degtyarev M.V. Structure and hardness of molybdenum upon deformation under pressure at room and cryogenic temperatures. International Journal of Refractory Metals and Hard Materials, 2014, vol. 43, pp. 59-63. DOI: 10.1016/j.ijrmhm.2013.10.022

12. Panfilov P., Ermakov Al., Antonova O., Pilyugin V. Plastic deformation of polycrystalline iridium at room temperature. Platinum Metals Review, 2009, vol. 53, pp. 139-144. DOI: $10.1595 / 147106709 X 463318$ 
Подана в журнал: 08.12.2017

УДК 620.179.1:620.162.4

DOI: $10.17804 / 2410-9908.2017 .6 .112-119$

\title{
ВЛИЯНИЕ БОЛЬШИХ ДЕФОРМАЦИЙ НА СТРУКТУРУ, ХАРАКТЕР РАЗРУШЕНИЯ И МЕХАНИЧЕСКИЕ СВОЙСТВА ТУГОПЛАВКИХ МЕТАЛЛОВ
}

В. П. Пилюгин*, Т. М. Гапонцева, Т. П. Толмачев, Л. М. Воронова, Т. И. Чащухина, М. В. Дегтярёв

\author{
Федеральное государственное бюджетное учреждение науки \\ Институт физики металлов имени М.Н. Михеева Уральского отделения Российской академии наук, \\ ул. С. Ковалевской, 18, Екатеринбург, Российская Федераџия \\ *Ответственный автор. Электронная почта: pilyugin@imp.uran.ru \\ Адрес для переписки: 620049, Екатеринбург, ул. Комсомольская, 34, Российская Федерация \\ Тел.: +7 (343) 378-38-05
}

В современном материаловедении бурно развивается область получения и исследования объемных субмикро- и нанокристаллических материалов деформационными методами под давлением. К ним относится прежде всего метод кручения (сдвига) под высоким квазигидростатическим давлением на вращаемых наковальнях Бриджмена. Метод позволяет проводить в материалах интенсивную (или мега-) пластическую деформацию без разрушения образцов.

В работе проведено исследование влияния давления на пластичность тугоплавких (или близким к тугоплавким) хрупких переходных $d$-металлов $\mathrm{V}, \mathrm{Cr}, \mathrm{Nb}, \mathrm{Ta}, \mathrm{Mo}, \mathrm{W}, \mathrm{Re}, \mathrm{Ir}$ при их деформации методом кручения под высоким давлением. Установлена величина кри-

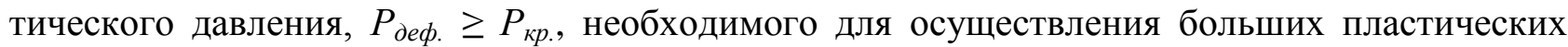
деформаций указанных металлов без их разрушения в схеме кручения на наковальнях. Необходимые для этого величины критического давления исследованных металлов составляют 0,05 от модуля объемной упругости или 0,015 от модуля сдвига. Структурные превращения тугоплавких малопластичных металлов, вызванные большой пластической деформацией, оказывают существенное влияние на их механические свойства, в частности достижение наноструктурного состояния этих металлов в результате воздействия кручением под высоким давлением выше установленной пороговой величины давления, обеспечивают значительное деформационное упрочнение по данным микротвердости. По данным фрактографического анализа, переход из моно- в нанокристаллическое состояние приводит к смене механизма разрушения образцов с хрупкого излома, сколом, на квазивязкое (вязкоподобное) разрушение образца по границам кристаллитов.

Ключевые слова: структура, интенсивная (мега-) пластическая деформация, высокое давление, критическое давление, тугоплавкие металль, пластичность, наноструктура, хрупкое разрушение, вязкое разрушение.

\section{1. Введение}

Переходные $3 d-5 d$-металлы имеют высокие значения энергии связи, упругих модулей (например для Мо: $\mathrm{E}=320$ ГПа; $\mathrm{G}=120$ ГПа; для $\mathrm{W}: \mathrm{E}=337$ ГПа; $\mathrm{G}=135$ ГПа, для Ir: $\mathrm{E}=570$ ГПа; $\mathrm{G}=220$ ГПа [1]), напряжений течения, коэффициентов деформационного упрочнения, а тугоплавкие $4 d-5 d$-металлы, как правило, характеризуются малым ресурсом пластичности и высокой хрупкостью. В жестких схемах испытаний с отрицательной или малой гидростатической компонентой напряжённого состояния относительное удлинение и относительное уширение для тугоплавких металлов, таких как тантал, монокристаллический иридий и вольфрам, составляют: $\delta_{\mathrm{Ta}}=30 \% ; \Psi_{\mathrm{Ta}}=75 \% ; \delta_{\text {Ir }}=6-10 \% ; \Psi_{\text {Ir }}=10-15 \% ; \delta_{\mathrm{W}}=0,6 \%$;

Pilyugin V.P. et al. / Effect of severe deformation on the structure, fracture pattern and mechanical proper-

ties of refractory metals 
$\Psi_{\mathrm{W}}=0 \%$ [1]. Их деформация без разрушения требует высоких температур $\left(\mathrm{T}_{\text {деф. }} \geq 0,8 \mathrm{~T}_{\text {плавл. }}\right)$, а при комнатной $\mathrm{T}_{\text {деф. }}=293 \mathrm{~K}$ и низких температурах необходима мягкая схема деформирования с высокой компонентой гидростатического сжатия [2]. Известно, что для деформации тугоплавких малопластичных металлов это давление должно быть достаточно высоким [2], но требует измерения конкретных значений для каждого из указанных металлов. Из всех методов статического деформирования давление такой величины достижимо только в безальтернативном по предельным величинам деформации методе кручения (сдвига) под давлением на наковальнях Бриджмена [3]. Существенно более высокие величины давлений достижимы только в ударно-волновых методах обработки, однако последние имеют ограничение по степени макродеформации. Известно, что давление пластифицирует материалы, позволяя получать большие деформации и вследствие этого субмикро- и наноструктурные состояния [4-7]. Это оказывает влияние на структурно-чувствительные фундаментальные и служебные свойства: фазовую стабильность, прочность, характер разрушения, трение и износ, тепло- и электропроводность и т. д. [8-10]. За последние 20-25 лет на эту тему выполнено большое количество работ, но они касались в основном $3 d$-переходных металлов и сплавов на их основе, в то время как тугоплавкие $d$-переходные металлы остаются мало исследованными при больших деформациях. Цели данной работы - определение величины критического давления, необходимого для деформации без разрушения образцов малопластичных тугоплавких металлов; изучение влияния микроструктурных изменений, вызванных большой деформацией под давлением на упрочнение и характер излома хрупких тугоплавких $d$-металлов.

\section{2. Материалы и методика}

Моно- и поликристаллические образцы металлов $\mathrm{V}, \mathrm{Cr}, \mathrm{Nb}, \mathrm{Ta}, \mathrm{Mo}, \mathrm{W}, \mathrm{Re}, \mathrm{Ir}$, чистотой не ниже ЧДА (т. е. чистый для анализа) подвергали деформации методом кручения (сдвига) под давлением в диапазоне 8-18 ГПа в наковальнях Бриджмена на гидропрессе максимальным усилием до $10^{6} \mathrm{H}$. Величину истинной деформации рассчитывали по формуле из [4], которая в настоящей работе изменялась от начальных $e=0,2$ до высоких $e=4,0-6,0$. При обработках образцов применяли металлокерамические сверхтвердые пуансоны из ВК-6 и кубического нитрида бора с-BN с диаметром рабочих площадок $2 R=5,0$ мм и $2 R=3,0$ мм соответственно. Дисковые образцы указанных металлов имели исходную толщину 0,25-0,35 мм и диаметр, соответствующий рабочим площадкам пуансонов наковален. Структурные измерения проводили методами металлографии, просвечивающей и сканирующей электронной микроскопии на ПЭМ JEM-200 CX и СЭМ Quanta-200 Pegasus, измерение микротвёрдости проводили на приборе ПМТ-3 [6]. Увеличение давления приводило к возрастанию неопределенности его значений вследствие ряда аппаратурных эффектов, например упругопластической деформации, как образцов, так и пуансонов.

\section{3. Результаты и их обсуждение}

Обработки серий образцов с различными усилиями сжатия гидропресса $F$ и, соответственно, давлений $P_{\text {деф. }}=F / S$ позволили определить значения давления $P_{\text {деф. }} \geq P_{\kappa p}$, необходимое достижение или превышение которого позволяло деформировать образцы каждого металла без их разрушения и проскальзывания на пуансонах на значительные величины. Здесь $S=\pi R^{2}$ - площадь рабочих площадок пуансонов. В итоге, для каждого из металлов было получено значение $P_{\kappa p}$, которые приводятся в таблице.

Под значительными величинами подразумевается такая истинная деформация, при которой в металле происходит формирование структуры, состоящей из однородных взаимно разориентированных на большие углы нанокристаллитов размерами менее 100 нм. Для этого необходима деформация не менее чем в $e=4-6$, что соответствует 1-5 полным оборотам пуансонов. При этом в результате интенсивной деформации в металлах развиваются процессы

Pilyugin V.P. et al. / Effect of severe deformation on the structure, fracture pattern and mechanical properties of refractory metals 
структурной фрагментации, что отражается на сопротивлении металлов деформированию и значениях их микротвёрдости (таблица).

Механические свойства исследуемых металлов

\begin{tabular}{|c|c|c|c|c|c|}
\hline Металл & $\begin{array}{c}\text { Модуль } \\
\text { объемной } \\
\text { упругости } \\
K, \text { ГПа }\end{array}$ & $\begin{array}{c}\text { Модуль } \\
\text { сдвига } \\
G, \text { ГПа }\end{array}$ & $\begin{array}{c}\text { Микротвердость } \\
\text { исходная, } \\
\text { МПа }\end{array}$ & $\begin{array}{c}\text { Микротвердость } \\
\text { после большой } \\
\text { деформации, } \\
\text { МПа }\end{array}$ & $\begin{array}{c}\text { Давление } \\
\text { деформации } \\
\text { без разрушения } \\
P_{\kappa p .}, \text { ГПа }\end{array}$ \\
\hline $\mathrm{V}$ & 139,42 & 47,3 & 650 & 3250 & $8,0 \pm 0,5$ \\
\hline $\mathrm{Nb}$ & 144,2 & 37,5 & 836 & 4430 & $7,5 \pm 0,5$ \\
\hline $\mathrm{Ta}$ & 201,9 & 70 & 2310 & 7920 & $11 \pm 1$ \\
\hline $\mathrm{Cr}$ & - & 110 & 1420 & 4100 & $12 \pm 1$ \\
\hline Mo & 253,1 & 120 & 2020 & 7650 & $14 \pm 2$ \\
\hline $\mathrm{W}$ & 300,09 & $125-155$ & 3480 & 15400 & $16 \pm 2$ \\
\hline $\operatorname{Re}$ & 475 & - & 2500 & 12400 & $18 \pm 2$ \\
\hline Ir & 649 & $209-220$ & 1960 & 15100 & $16 \pm 2$ \\
\hline
\end{tabular}

При $P_{\text {деф. }} \geq P_{\kappa p}$. образование трещин наблюдается только в периферийной части образцов хрупких металлов (Cr, Mo, W, Ir, Re), где давление ниже среднего квазигидростатического. Как правило, образование периферийных трещин происходит при декомпрессии, и их образование при снижении давления регистрировалось по акустической эмиссии, а после извлечения образцов из камеры Бриджмена - визуально и металлографически. Деформация под давлением $P_{\text {деф. }}<P_{\kappa p}$. на наковальнях сжатием, кручением и последующая разгрузка приводят к появлению катастрофических трещин разрушения, устьем направленных к центру дисковых образцов и приводящих к их распаду на отдельные части.

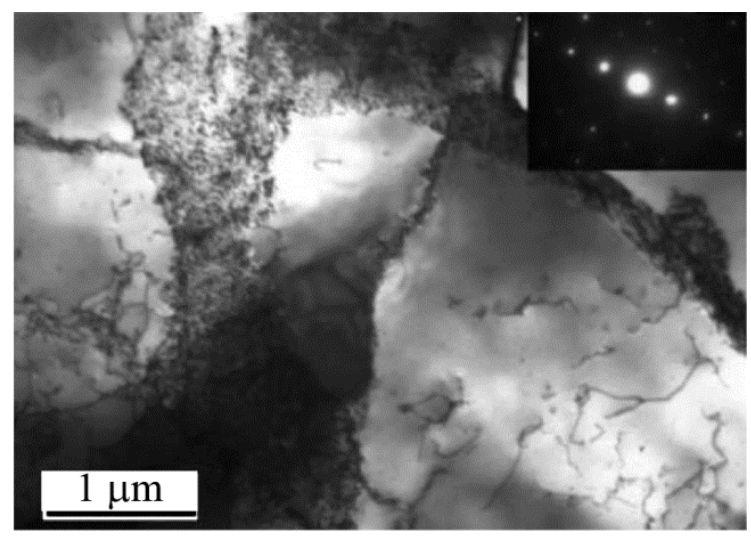

$a$

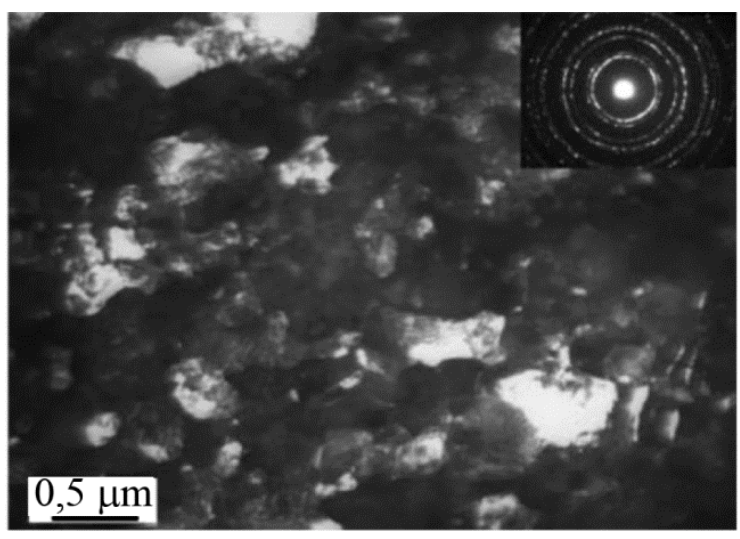

$\sigma$

Рис. 1. Микроструктура и электронная микродифракция иридия: $a$ - исходное состояние; $\sigma$ - нанокристаллическое состояние после деформации $e=5$ при $P=15$ ГПа, темное поле в рефлексе (111)

Для объяснения механизма барической пластификации металлов необходимо отметить, что появление трещин разрушения связано с зарождением, движением дислокаций и образованием дислокационных скоплений. При этом необходимым условием нарушения сплошности в момент появления трещин разрушения является наличие растягивающих напряжений в скоплениях. Общим, и очевидным при этом выводом является то, что приложение высокого внешнего гидростатического давления уменьшает растягивающие напряже-

Pilyugin V.P. et al. / Effect of severe deformation on the structure, fracture pattern and mechanical proper- 


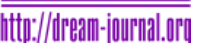

ния в деформируемом теле и тем самым подавляет процесс зарождения трещин разрушения. Установленные значения давлений $P_{к p}$. для исследованных металлов составляют $K / 20(0,05$ от модуля объемной упругости) или $G / 60$ (около 0,015 от модуля сдвига металла).

Процессы эволюции микроструктуры переходных $3 d$-металлов ранее подробно исследованы в ряде работ [4-7]. Большая деформация свыше 3-4 единиц вызывает активную фрагментацию структуры исходных моно- и поликристаллических металлов. Для иридия, рения и вольфрама средний размер кристаллитов составляет не более 20-30 нм (рис. 1). Отметим, что в конечном структурном состоянии тугоплавкие металлы, особенно $5 d$, таких как Ir, $\mathrm{W}, \mathrm{Re}$, имеют качественное отличие от менее тугоплавких $d$-металлов. Это выражается в форме и в размерах кристаллитов - в структуре тугоплавких металлов форма кристаллитов не столь равноосная, распределение их по размерам имеет больший разброс и имеется внутренняя субструктура кристаллитов (рис. 1.). Это связано с меньшей интенсивностью процессов возврата и релаксации в тугоплавких металлах в силу более высоких значений их температур плавления и соответственно более низкой гомологической температуры деформирования $\mathrm{T}_{\text {гом. }}=\mathrm{T}_{\text {деф. }} / \mathrm{T}_{\text {пл. }}[11,12]$.

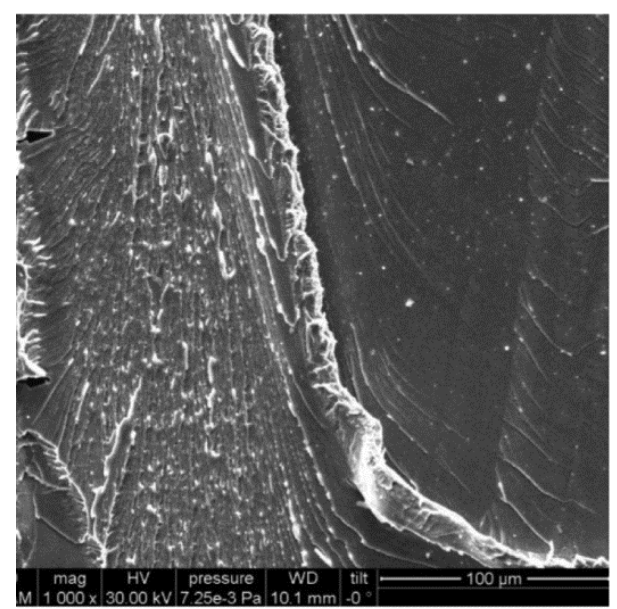

$a$

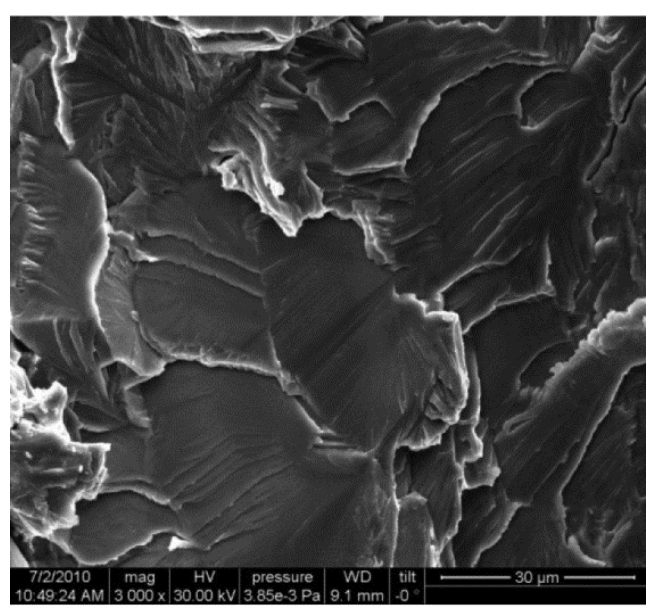

$\sigma$

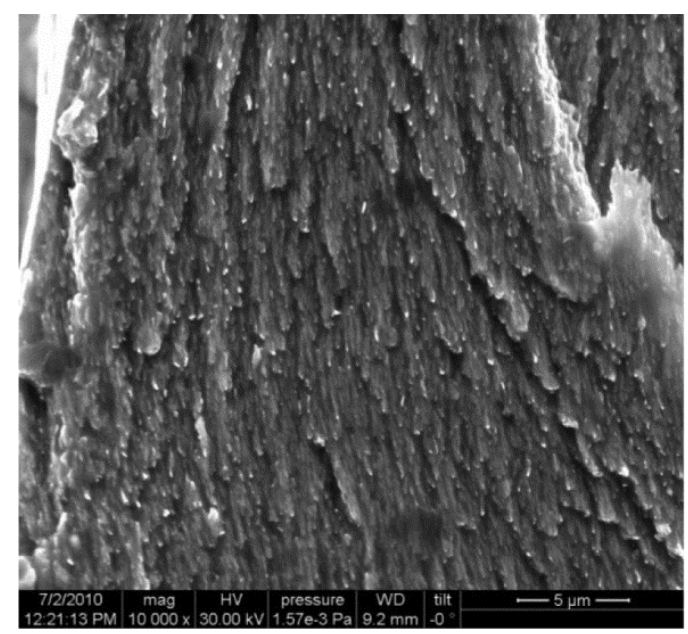

B

Рис. 2. Поверхности изломов образцов молибдена на середине радиуса образцов: $a$ - хрупкое разрушение исходного монокристалла Мо (стрелками отмечена область хрупкого разрушения); $\sigma$ - хрупкое разрушение фрагментированного вследствие деформации Мо $e=0,7$ при $P=15$ ГПа; $в-$ вязкоподобное разрушение нанокристаллического Мо, $e=5,5$ при $P=15$ ГПа

Pilyugin V.P. et al. / Effect of severe deformation on the structure, fracture pattern and mechanical properties of refractory metals 
Большая пластическая деформация оказывает влияние на структурно зависимые физико-механические свойства металлов, следовательно, как нами установлено, большая пластическая деформация тугоплавких металлов приводит к смене характера разрушения от хрупкого к хрупко-вязкому вследствие высокой деформационной фрагментации структуры. Выявлено изменение характера излома от хрупкого транскристаллитного в моно- и поликристаллических металлах на вязкоподобный межкристаллитный. По мере фрагментации исходной крупнокристаллической структуры металлов и накопления в них участков взаимной разориентировки при потере монокристаллами ориентационной устойчивости начинает сказываться влияние дефектов на характер разрушения. Пример влияния фрагментации на характер разрушения исходно монокристаллических образцов молибдена представлен на рис. 2. Аналогичный эффект проявляется и в других исследованных металлах, хрупком хроме, а также тугоплавких и хрупких металлах - рении, иридии и вольфраме (рис. 3).

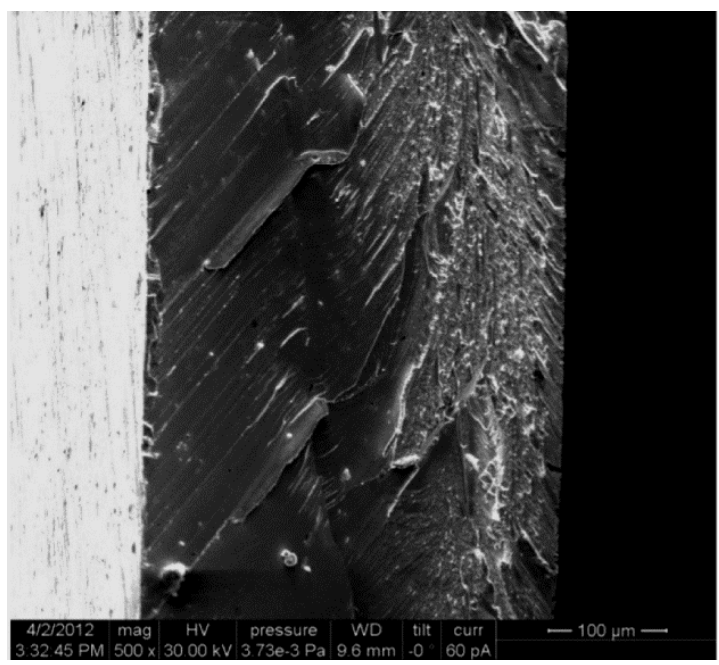

$a$

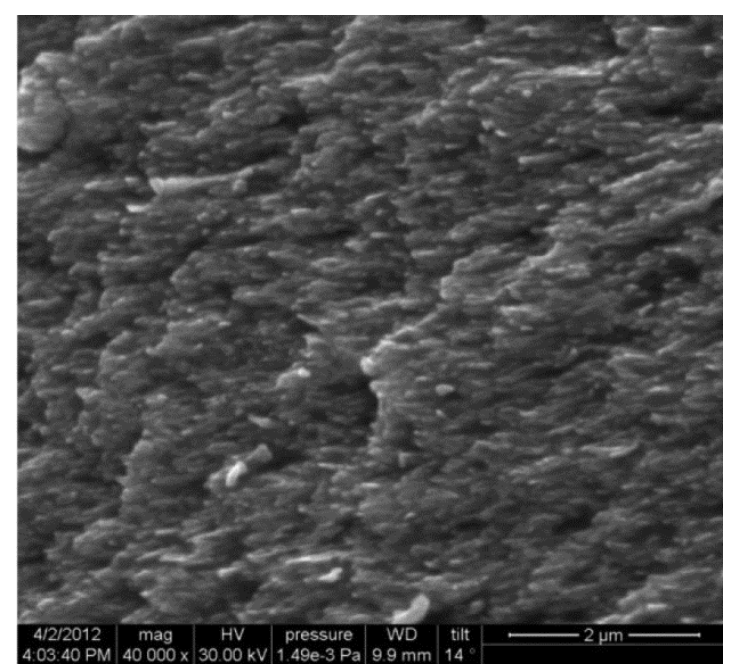

$\sigma$

Рис 3. Хрупкий скол монокристаллического образца вольфрама $(a)$,

вязкоподобное (б) разрушение сильнодеформированного нанокристаллического образца вольфрама $e=4,8 ; P=18$ ГПа. Фрактограммы получены на середине радиуса образцов

Механизм перехода от хрупкого разрушения к вязкоподобному при переходе от структурного монокристаллического состояния в нанокристаллическое для хрупких металлов обусловлен сменой механизмов деформации с дислокационного скольжения в монокристаллах на доминирующее зернограничное (межкристаллитное) проскальзывание, присущее нанокристаллическим структурам.

\section{4. Заключение}

Установлена величина критического давления, необходимого для осуществления больших пластических деформаций тугоплавких металлов без их разрушения в схеме кручения (сдвига) под давлением. Величина давления составляет не ниже 0,05 от модуля объемной упругости или 0,015 от модуля сдвига металла. Структурные превращения металлов, вызванные большой деформацией с образованием субмикро- и наноструктурного состояния оказывают существенное влияние на механические свойства металлов, приводя к высокому деформационному упрочнению и смене механизма хрупкого разрушения сколом на квазивязкое (вязкоподобное) по границам нанокристаллитов (нанозёрен).

Pilyugin V.P. et al. / Effect of severe deformation on the structure, fracture pattern and mechanical proper- 


\section{Благодарность}

Работа выполнена в рамках государственного задания ФАНО России (тема «Деформация» при частичной поддержке проекта УрО РАН № 15-17-2-11. Электронномикроскопическое исследование выполнено в ЦКП «Испытательный иентр нанотехнологий и перспективных материалов» ИФМ УрО РАН.

\section{Литература:}

1. Element Properties : reference work / ed. by Drits M.E. - M. : Metallurgiya, 1985. 672 pages. (In Russian).

2. Frost H. J., Ashby M. F. Deformation-mechanism maps. The plasticity and creeps of metals and ceramics. - Cambridge University, UK : Pergamon press, 1982. - $166 \mathrm{p}$.

3. Bridgman P. W. Effects of High Shearing Stress Combined with High Hydrostatic Pressure // Physical Review. - 1935. - Vol. 48. - P. 825-847.

4. Structure evolution of pure iron upon low-temperature deformation under high pressure / V. P. Pilyugin, L. M. Voronova, M. V. Degtyarev, T. I. Chashchukhina, V. B. Vykhodets, T. E. Kurennykh // The Physics of Metals and Metallography. - 2010. - Vol. 110, no. 6. - P. 564573. - DOI: 10.1134/S0031918X10120070

5. Evolution of the structure and hardness of nickel upon cold and low-temperature deformation under pressure / V. P. Pilyugin, T. M. Gapontseva, T. I. Chashchukhina, L. M. Voronova, L. I. Shchinova, M. V. Degtyarev // The Physics of Metals and Metallography. - 2008. - Vol. 105, no. 4. - P. 409-419. - DOI: 10.1134/S0031918X08040157

6. Influence of the relaxation processes on the structure formation in pure metals and alloys under high-pressure torsion / M. V. Degtyarev, T. I. Chashchukhina, L. M. Voronova, A. M. Patselov, V. P. Pilyugin // Acta Materialia. - 2007. - Vol. 55, no. 18. - P. 6039-6050. DOI: 10.1016/j.actamat.2007.04.017

7. Structural Changes and Properties of Molybdenum upon Cold and Cryogenic Deformation under Pressure / T. M. Gapontseva, V. P. Pilyugin, M. V. Degtyarev, L. M. Voronova, T. I. Chashchukhina, A. M. Patselov // Russian Metallurgy (Metally). - 2014. - No. 10. - P. 812816. - DOI: 10.1134/S0036029514100024

8. Thermal diffusivity of submicro- and nanocrystalline niobium, titanium, and zirconium at high temperatures / V. I. Gorbatov, V. F. Polev, V. P. Pilugin, I. G. Korshunov, A. L. Smirnov, S. G. Talutz, D. A. Brytkov // High Temperature. - 2013. - Vol. 51, no. 4. - P. 482-485. DOI: $10.1134 / \mathrm{S} 0018151 \mathrm{X} 1304010 \mathrm{X}$

9. The Influence of Nanocrystalline Structure of Iron on 6-e Phase Transformations under Pressure / Vitaly Pilyugin, Alexander Patselov, Timofey Tolmachev, Eugeny Chernyshov, Alexey Ancharov, Dmitriy Brytkov // Material Science Forum. - 2013. - Vol. 738-739. - P. 108-113. DOI: 10.4028/www.scientific.net/MSF.738-739.108

10. Koroleva L. F. Nanoparticulate zirconia-modified solid solutions of aluminum-iron oxides for polishing titanium metal // Diagnostics, Resource and Mechanics of materials and structures. 2015. - Iss. 1. - URL: http://dream-journal.org/DREAM_Issue_1_2015_Koroleva_L._F..pdf

11. Structure and hardness of molybdenum upon deformation under pressure at room and cryogenic temperatures / V. P. Pilyugin, L. M. Voronova, T. M. Gapontseva, T. I. Chashchukhina, M. V. Degtyarev // International Journal of Refractory Metals and Hard Materials. - 2014. Vol. 43. - P. 59-63. - DOI: 10.1016/j.ijrmhm.2013.10.022

12. Panfilov P., Ermakov Al., Antonova O., Pilyugin V. Plastic deformation of polycrystalline iridium at room temperature // Platinum Metals Review. - 2009. - Vol. 53. - P. 139-144. DOI: $10.1595 / 147106709 X 463318$ 